\title{
Crise Nationale et Crise Spatiale en Somalie ${ }^{1}$
}

\author{
Alain Gascon
}

La Somalie est l'Etat le plus homogène qui soit en Afrique au Sud du Sahara, hormis la minorité bantoue et les locuteurs du swahili de la côte, le somali est parlé par tous et tous professent l'Islam shaf'îte. Tous les auteurs insistent sur le modèle social que représente l'éleveur nomade, modèle qui prévaut encore en dépit de l'exode rural. Leurs travaux révèlent la richesse du patrimoine poétique partagé par tous les Somali.

Pourtant, cette unité n'a pas empêché les déchirements quel'on sait, longtemps mis sur le compte du partage colonial. Or, la chute de la "maison" Siyaad a entraîné la Somalie : "Nation in Search of a State" (Laitin \& Samatar, 1987) dans un éclatement tel, qu'il faudrait maintenant plutôt dire : "Nation in Search of Herself". Maintes explications ont été données : les clans et les nomades "instables", la crise, le réveil de l'Islam... Aucune, cependant, ne tient compte de la gestion de l'espace dans la reproduction des systèmes sociaux de production pastorale et agro-pastorale : quel est le rapport à l'espace des Somali, quel espace produit une société d'éleveurs nomades et d'agro-pasteurs en crise ? Cet article tente de répondre à cette question.

\section{Une forte unité?}

On sait par expérience que l'usage d'une langue commune est un critère ni suffisant ni nécessaire à l'unité nationale: Renan et Mommsen en débattaient déjà au siècle dernier à propos de l'AlsaceLorraine de langue germanique, mais partie intégrante ${ }^{2}$ de la nation française. A l'hostilité des partisans de l'usage de l'arabe, s'est ajouté le scandale de l'utilisation de l'alphabet latin pour écrire le somali (D.D. Laitin \& S.S. Samantar, 1987).

Alain Gascon, géographe, Laboratoire de Sociologie et de Géographie Africaines, Centre National dela Recherche Scientifique, Paris, France.

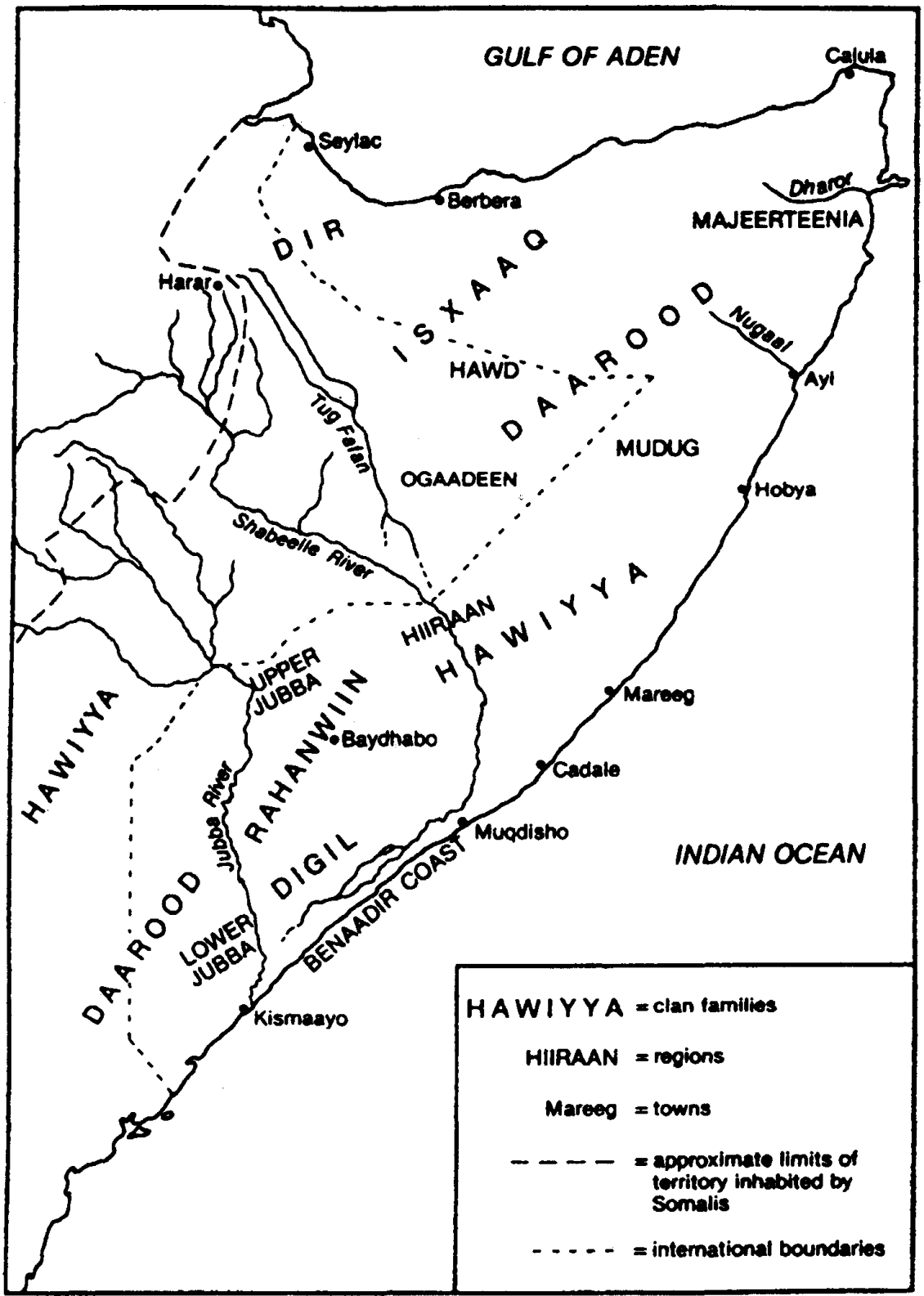

Fig. 1: The Horn of Africa: Present distribution of Somali clan-families (From: L. V. Cassanelli)

La rivalité entre les confréries (Qadiriya, Ahmediya, Salihiya) divise profondément l'unité "mystique" de l'Islam somali (I.M. Lewis, 1982), les saints fondateurs étant assimilés aux ancêtres éponymes des clans.
La péninsule somali est incluse d'un bloc dansle Sahel; trop souvent on oublie que Mugdisho està la latitude du Gabon, Sahel certes, mais Sahel équatorial ! Il faut introduire une double opposition entre la "Somalie heureuse" 3 du Nord, 
rebord vigoureux du Rift d'Aden qui dépasse $2000 \mathrm{~m}$, surplombe une tihama ${ }^{4}$ torride (guban) et reçoit plus de $750 \mathrm{~mm}$ de précipitations, le plateau aride du Hawd et la Mésopotamie somalienne où la courbe des pluies se relève vers le Sud.

Contrairement au Sahara, l'eau est accessible, notamment au pied des failles et le long des deux fleuves pérennes qui descendent des hauts plateaux $\mathrm{d}^{\prime}$ Ethiopie, le Jubba et le Wabi Shabeele, le Nil de Mugdisho qui coule parallelement à la côte sur $400 \mathrm{~km}$ au Benaadir : une Egypte avec deux Nils ! Manquant de longues séries pluviométriques, on pense que les récurrences sèches reviennent tous les dix ans avec des minima inter-stadaires tous les trois ou quatre ans (L.V. Cassanelli, 1982). Peuton étendre à toute la péninsule la succession admise de quatre saisons : une saison sèche courte, xaaga, coupant la saison humide en deux sous-saisons humides, gu et dayr, s'opposant à la longue saison sèche, jillaal?

\section{Les clans et les formes d'occupation de l'espace}

Ce milieu naturel fragile où règne la précarité, est partagé selon la segmentation lignagère jusqu'à l'unité élémentaire de lignage, le reer, croisé avec les pactes territoriaux, les xeer (Fig. 1). Ces alliances temporaires regroupent des groupes voisins issus de clans différents qui s'engagent à défendre les pâturages et à payer le prix du sang. Ils sont conclus dans des assemblées, les shir, dont tous les membres sont égaux en droits et en devoirs : I.M. Lewis y identife l'institution fondamentale de la "pastoral democracy" qui régit les Somali. Au gré du rythme des précipitations, l'espace résidentiel, les hameaux enclos ou gurri, et l'espace technique, les parcours,sont redécoupés chaque année. Pour ma part, pour rendre compte de cette situation, je préfèrerais employer l'expression "anarchie pastorale"

Les clans étaient aussi des instruments de gestion de l'espace de telle façon que la structure sociale fût reproduite. L.V. Cassanelli décrit fort bien la complémentarité ville-campagne qui fonctionnait en cas de sécheresse: les "bouches inutiles" étaient placées chez les parents dans les ports, les petits ruminants puis les bovins étaient sacrifiés pour acheter la nourriture à l'etranger afin de conserver aussi longtemps que possible une présence auprès des puits et des pâturages pour les réinvestir une fois la crise passée. Il remarque que les sécheresses coïncident avec un accroissement sensible des exportations d'animaux qui transparaît dans les séries statistiques coloniales italiennes.

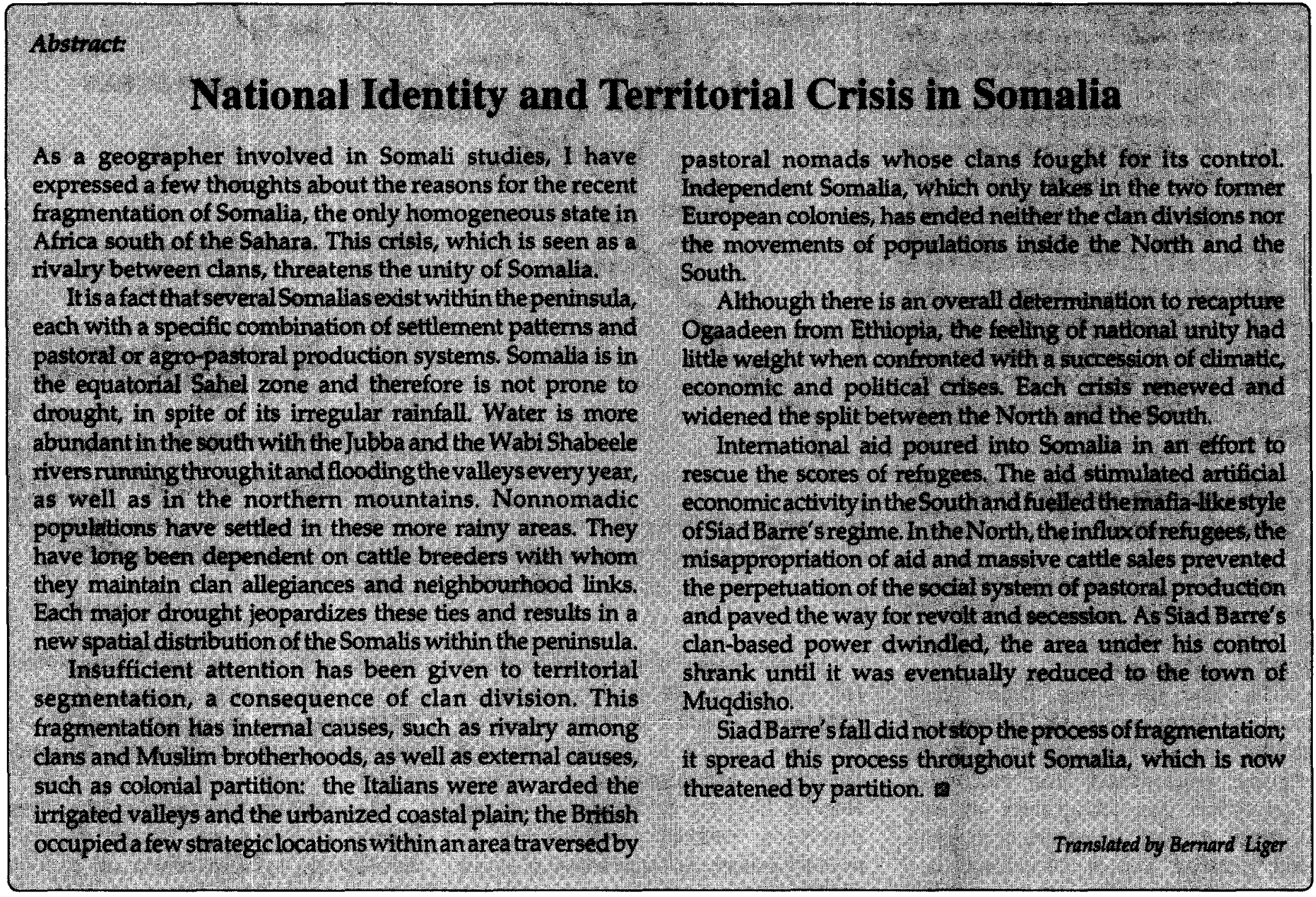

Refuge, Vol. 12, No. 5 (November-December 1992) 
Après l'épreuve, une nouvelle répartition des parcours s'instaurait dans la violence. Pendant l'été, pendant les pluies, les hommes migraient vers les pâturages du Hawd disputés entre Isaaq et Ogaadeen 6 (J. Markakis, 1989). Une autre complémentarité territoriale se formait avec les agriculteurs sédentaires des plateaux du Nord et des vallées et des mares du Sud, par le biais de la solidarité et de la sujétion claniques (Fig. 2).

On peut esquisser une géographie des formes d'occupation de l'espace au siècle dernier qui s'est prolongée jusqu'aux crises récentes. La Somalie des puits de clans, ceel, et des réservoirs, basked, correspond au Nord, au Nogaal et à l'Ogaadeen. La Mésopotamie et le Benaadir sont le domaine de villages d'agriculteurs égrennés le long des fleuves, parmi les forêts-galeries peuplées de Bantous, chasseurs, pêcheurs et cultivateurs temporaires, dans le bas Jubba. Autour de lacs temporaires de dérivation des hautes eaux, deshek, ou de mares pluviales, war ou baali, la culture de décrue fixe de gros villages (Fig. 3).

Au siècle dernier, les bénéfices de la vente des carcasses aux colonies européennes étaient réinvestis dans des plantations de coton, irriguées par des canaux dérivés de barrages sur le bas Wabi Shabeele. Elles étaient cultivées à l'aide d'esclaves achetés à Zanzibar. Une bande densément humanisée, perpendiculaire puis parallèle à la côte, formait l'hinterland du chapelet des ports du Benaadir, déjà intégré au marché international.

\section{Les dynamiques spatiales et le partage colonial}

La péninsule somali était déjà soumise à des formes économiques d'organisation de l'espace différentes et divergentes, avant le partage colonial.

Au Nord, un espace "fluide" était animé par les mouvements "browniens" autour des puits; et chaque variation du régime engendrait une nouvelle répartition des troupeaux et des hommes. Au Sud, un espace homogène était "polarisé" vers la côte extravertie.

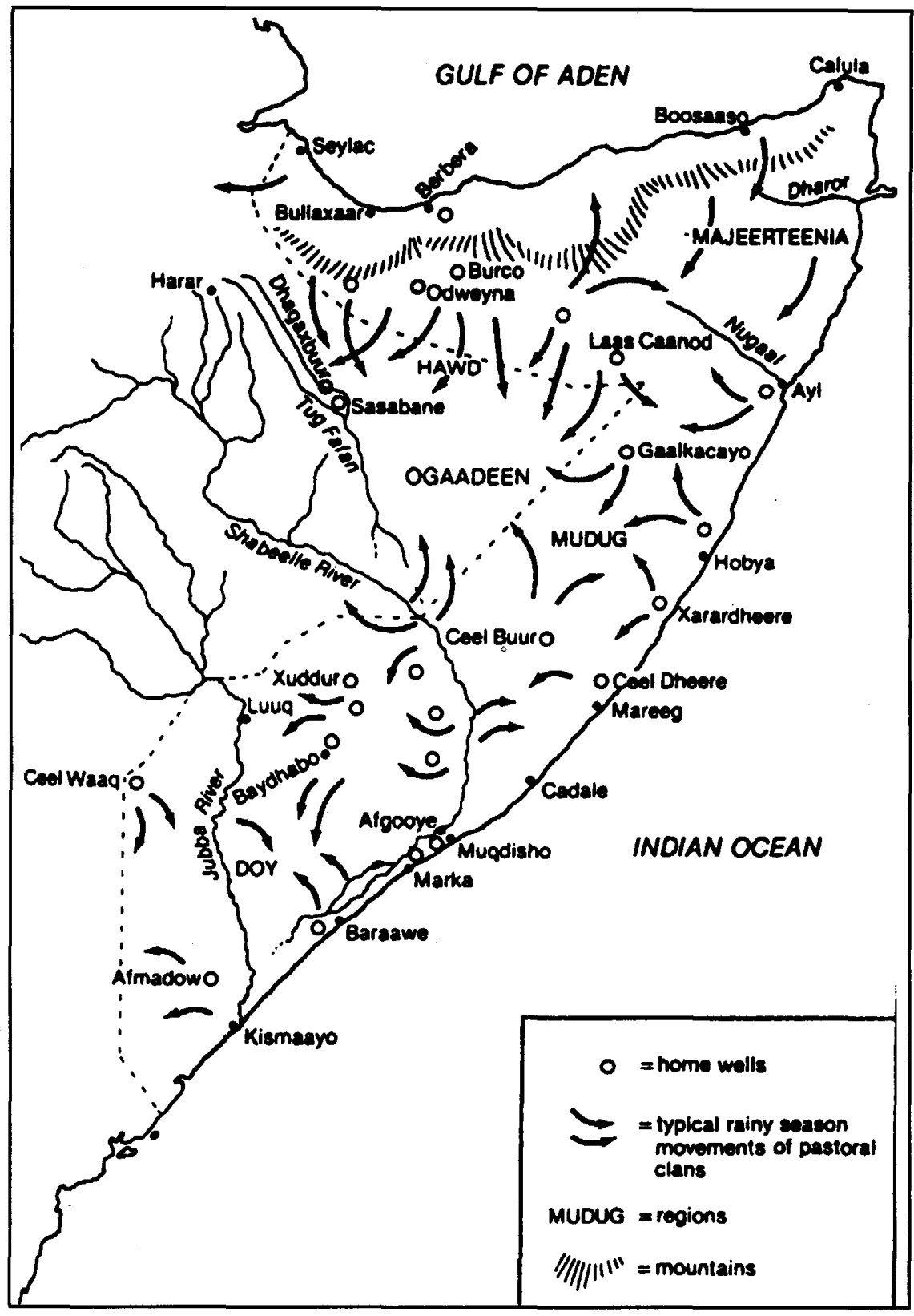

Fig. 2: Major patterns of Somali transhumance (twentieth century) (From: L. V. Cassanelli)

La colonisation coïncida, engendra et fut également la conséquence de mouvements internes à la Corne de l'Afrique comme le prosélytisme des confréries musulmanes. Aw Awees, sheykh Uways, de Baraawe et ses disciples, wadaad, installèrent de nombreuses zawiya ou jamaha autour des tombeaux des saints. Elles furent particulièrement denses dans la Mésopotamie somalienne chez les cultivateurs hors-clans (C. Ahmed, 1989).

Les raids entrepris par les Ethiopiens après la prise de Harär par ras Mäkonnen, agirent comme un détona- 
teur et firent voler en éclats le partage du territoire entre les clans-familles, segmentation territoriale et segmentation clanique demeurant intimement liés (H.S. Lewis, 1964). La jihad de sayyid Maxamed Cabdulle Xasan fut dirigée à la fois contre les gaal, les Européens, mais aussi contre ses ennemis de clans et de confréries (C. Ahmed, 1989). Jouant des rivalités coloniales, le sayyid se fixa à Taleh, une ville forteresse située à proximité des limites des zones d'influence des conquérants.

Le partage n'entrava les déplacements des éleveurs qu'après le retour de l'Ogaadeen et du Hawd à l'Ethiopie en 1955. Italiens et Britanniques autorisaient la transhumance vers le Hawd alors que les Ethiopiens tentaient de la contrôler afin de percevoir des impôts et de limiter les infiltrations de guérilleros dans le "glacis" du Harär, après l'indépendance

\section{Les sécheresses récurrentes, la guerre, la croissance démographique, l'explosion urbaine et l'intégration au marché international agirent comme un révélateur des déséquilibres entre les régions et les aggravèrent.}

de la Somalie en 1960 (J.Markakis, 1987).

Jusqu'au milieu des années 1970, les camions qui se rendaient de Hargeysa versleSud, coupaient nuitamment parla voie directe, l'Ogadén en territoire éthiopien, en toute impunité.

Les projets des puissances européennes étaient différents. Les Anglais furent entraînés dans la colonisation pour déloger les Français installés à Seylac en face d'Aden. Ils s'engagèrent dans la guerre contre le sayyid pour ne point perdre la face. Ils maintinrent une présence militaire minimaleà partir des deuxports, Berbera et Seylac et d'une ville à l'intérieur, Hargeysa?

Pour les Italiens, la côte de la Somalie était le tremplin dela conquête future des hautes terres salubres d'Ethiopie. Maîtres du Benaadir puis du bas Jubba, du littoral urbanisé et de l'arrière-pays fertile mis en valeur par une population dense d'agriculteurs, les Italiens remontèrent le cours des deux fleuves, délaissant la Majerteen et le Nogaal d'ailleurs traversés par la frontière du Somaliland. Ilsne développèrent pasune colonie de peuplement, mais découpèrent de grandes plantations cotonnières, sucrières et bananières sur le bas Wabi Shabeele (Genale, Villabruzzi, maintenantJowhar) pourles besoins de la métropole.

Hormis les cadres européens, la main-d'oeuvre était indigène, salariés permanents et ouvriers temporaires réquisitionnés. En proportion, la population italienne de Mugdisho était beaucoup moins nombreuse que celle d'Asmära sur les plateaux d'Erythrée ! Endehors de cetterégion au Nord-Est du Wabi Shabeele, le seul établissement italien important était les salines de RasHafun, enclave extravertie vers l'exportation, en liquidationjudiciaire en 1937.

La même évolution divergente, la même dynamique spatiale contradictoire se poursuivit jusqu'à l'indépendance, sous la tutelle de $l^{\prime}$ Administration Fiduciaire Italienne de la Somalie ${ }^{8}$ au Sud et des Anglais au Nord.

\section{Les dynamiques spatiales et la crise des systèmes sociaux de produc- tion pastorale et agro-pastorale}

Cette crise resta sous-jacente jusqu'à la Révolution de 1969, jusqu'à l'instauration sous la férule de Siyaad Barre, d'un Etat fort, centralisé, nationaliste et se réclamant du marxisme-léninisme modernisateur et niveleur de toutes les traditions qui assuraient l'articulation entre l'espace résidentiel et l'espace technique. Les sécheresses récurrentes, la guerre, la croissance démographique, l'explosion urbaine et l'intégration au marché international agirent comme un révélateur des déséquilibres entre les régions et les aggravèrent. Alors que le gouvernement et les organisations internationales de développement prétendaient agir dans le but de réduire les écarts régionaux, les éleveurs du Centre et du Nord ressentirent les mesures d'aide impulsées depuis la capitale commme un complot visant à les affamer par la mise en défens de périmètresirrigués de plusen plus vastes et à les assujettir par les programmes de sédentarisation. La dérive maffieuse du pouvoir central ne fit que confirmer ces accusations.

\section{La "Révolution" de 1969 et les dynamiques spatiales}

L'indépendance n'entraîna pas de rupture radicale dans les dynamiques spatiales mises en oeuvre pour la reproduction des systèmes sociaux de production. Les plantations bananières, cotonnières et sucrières du Benaadir exportaient largement vers le marché de l'ancienne métropole. De grandes sociétés italiennes implantées depuis l'avant-guerre continuaient la tradition coloniale. De petits exploitants somaliens pratiquaient une polyculture irriguée à laquelle ils avaient intégré des cultures de rente. Une industrie de transformation des produits agricoles s'était installé à Mugdisho et dans les environs.

La Révolution de 1969 instaura le socialismeet procéda à la nationalisation des plantations et des entreprises industrielles possédées par les étrangers (P. Decreane, 1977). Les exploitants somaliens furent enserrés dans un système de livraisons obligatoires. Des fermes d'Etat mécanisées sur le modß̉e des sovkhoz, tentèrent une céréaliculture sèchesurles plateauxdelaMésopotamie et du Nord en recourant à la mobilisation de la main-d'oeuvre "volontaire".

\section{L'impact spatial de la sécheresse}

Le bilan de la catastrophe de 1973-75 a été dressé par P.Decreane et T. Labahn, celui des crises plus récentes est plus difficile à établir dans le désordre croissant qui s'installa dans les dernières années de Siyaad. 
Le cheptel subit des pertes importantes et ce furent les régions du Nord qui furent le plus touchees. Les cultures irriguées du Sud souffrirent maisjamais les fleuves ne setarirent alors que les puits et les mares s'asséchèrent. L'agriculture sèche et le dry farming pratiqués au Centre et au Nord ne survécurent pas à la succession des crises climatiques?. La sécheresse Dhabaadheer (1973-75) n'a pas seulement touché les hommes (17000 morts, 300000 déplacés) mais aussi le cheptel: un tiers a péri (273 000 dromadaires, 4,4 millions de chèvres et de moutons et 289000 bovins). Cinq années d'investissements ont été perdues. Dans les années 1980-85, la Somalie importait les $2 / 3$ de sa nourriture (dont la moitié sous forme d'aide). Et la sécheresse frappa à nouveau en 1984-85 eten 1987 au Nord et au Centre.

La crise climatique atteignit certes une ampleur exceptionnelle et frappa un pays soumis à un fort accroissement de sa charge démographique; mais, la "détribalisation" avait affaibli le système clanique. Le gouvernement tenait également à montrer son efficacité et profita de l'occasion pour précipiter la modernisation des systèmes de production "archaïques" comme on le fit en 1984-85 en Ethiopie. La mesure d'urgence la plus spectaculaire fut le transfert autoritaire, par avion, de 300000 éleveurs vers le Sud. On les installa dans des villages sur les plateaux et dans les vallées où on les convia fortement à devenir agriculteurs. On tenta même la reconversion en pêcheurs, pour un petit nombre, néanmoins.

Les projets de développement visèrent en priorité à étendre les surfaces irriguées aux dépens de l'agriculture de décrue par la construction de retenues sur le Wabi Shabeele et sur le Jubba à Fanoole : on ne pouvait étendre l'irrigation qu'au Sud. Les périmètres furent consacrés en priorité aux cultures $d^{\prime}$ exportation et aux cultures industrielles qui alimentèrent de nouvelles usines de transformation (sucrerie de Jowhar, cotonnerie de Balcad) situées dans le Benaadir, à Mugdisho et à Kismaayo. Ces entreprises d'Etat réalisaient en fait des

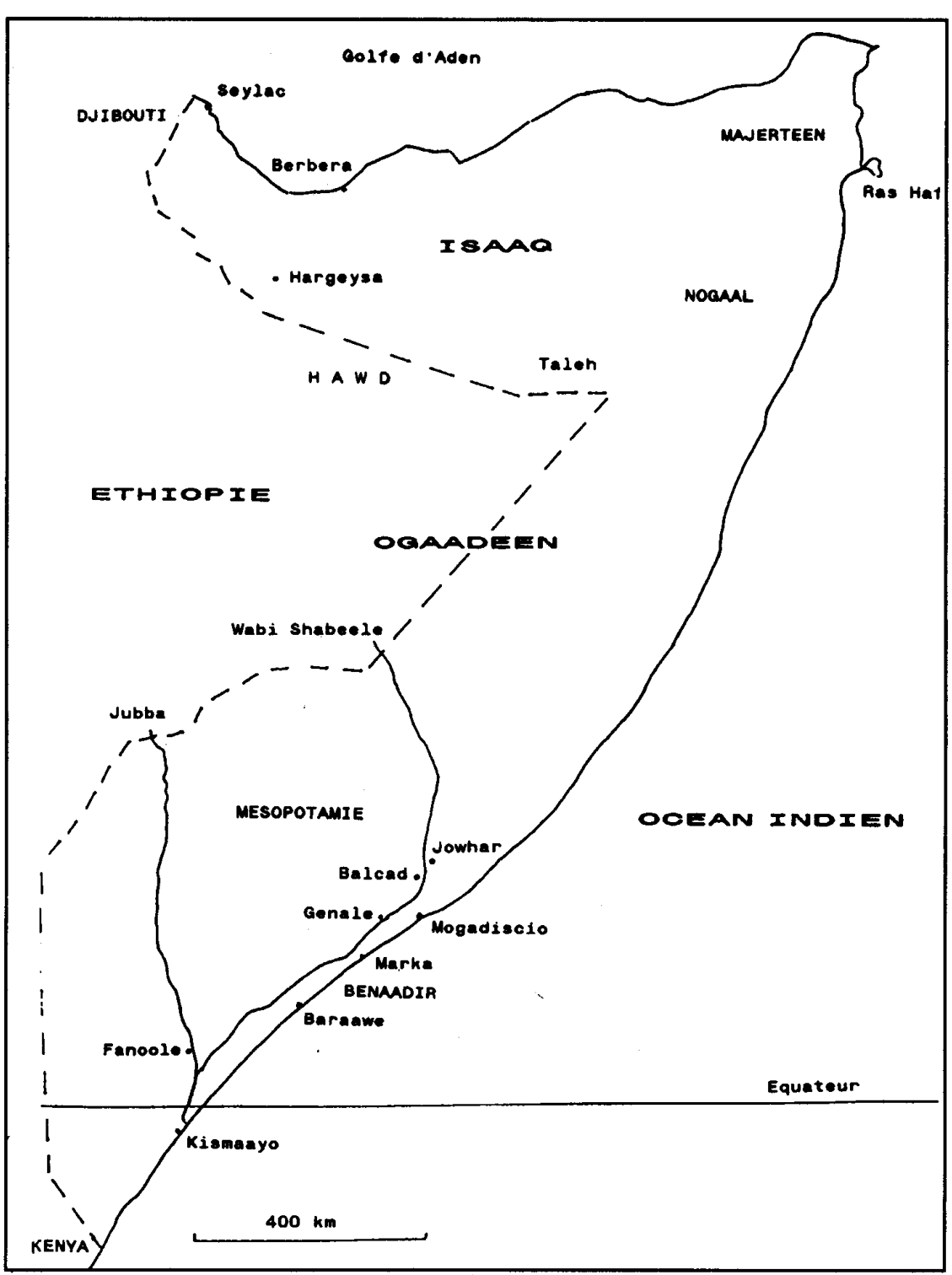

Fig. 3: Somalie

projets conçus pendant la période coloniale, mais financés par des capitaux étrangers, arabes surtout après l'adhésion à la Ligue Arabe, et exécutés par des techniciens des pays de l'Est.

L'Italie boudant les produits de Somalie depuis la nationalisation de ses intérêts, on chercha des débouchés en Arabie et dans les pays du Golfe (où vivait une forte diaspora somalienne). $\mathrm{La}$ part des exportations de bestiaux versles pays arabes augmenta jusqu'à atteindre
$90 \%$ du total à la fin des années 1970 . En 1983, l'Arabie invoquant des motifs sanitaires, diminua de moitié ses achats destinés au pélerins de la Mecque et plongea l'économie somalienne dans un marasme dont elle se releva incomplètement même après la reprise des achats séoudiens (J.Janzen, 1986; A.J. Gray, 1989).

Cette irruption brutale de l'économie de marché monétarisée parmi les éleveurs, rompit les solidarités 
traditionnelles déjà distendues et introduisit la compétition individuelle pour la maîtrise de l'espace technique par la multiplication des réservoirs bétonnés, enjeux d'affrontements sanglants au Nord et dans le Hawd entre Isaaq et Ogaadeen. (J.Markakis, 1989). Ces conflits préparèrent puis alimentèrent la rébellion du Mouvement National Somali dirigée et soutenue par les Isaaq ${ }^{10}$.

\section{La guerre aggrave la crise spatiale}

La guerre somalo-éthiopienne (1977-78) a gonflé le déficit, accumulé les pertes humaines et animales et entraîné un afflux de réfugiés. Le régime s'engagea dans une politique d'endettement qui le conduisit à passer sous la tutelle du FMI (la dette extérieure bondit de 1 milliard de $\$$ en 1980 à 1,8 milliard de $\$$ en 1987 et équivalait au PIB). L'industrie, déjà désorganisée par les nationalisations $s^{\prime}$ effondra et la Somalie couvrit de moins en moins ses importations. Deplus, 1 'aide internationale qui représenta $40 \%$ des ressources du budget somalien, était récurrences sèches, était moins favorable à leur établissement ; pourtant moins nombreux, leur poids y était insupportable.

Les camps de réfugiés jouent un rôle décisif dans la "citadinisation" des éleveurs. Les réfugiés ont été déplacés le plus souvent en camion, si ce n'est en avion, sur de longues distances et pour de longues périodes : les liens claniques se sont distendus et les pactes de voisinage, rompus. De plus, les camps présentent un habitat léger proche de l'habitat "spontané" des bidonvilles et sont organisés en quartiers qui les habituent à "consommer" des services qu'ils trouveront en ville. Ceux qui sortent des camps se dirigent naturellement vers les bidonvilles.

\section{Les réfugiés et le croît naturel submergent les villes}

La Somalie dans les frontières de $1938^{11}$, était peuplée de 1200000 habitants; quarante ans plus tard, $P$. Decreane et J. Janzen estimaient la population à plus de

\section{Le népotisme érigé en système de gouvernement et de prédation entraîna une appropriation familiale de l'Etat, sa patrimonialisation. Sa chute brisa même le seul lien qui unissait l'opposition.}

distribuée à partir de la capitale et parvenait au Nord non seulement en retard, mais diminuée par les détournements. Cette manne fut pourlaSomalie, toute proportion gardée, ce quela manne pétrolière fut pour les Etats du Golfe; elle acheva de désorganiser l'économie et les relations sociales. Le pays s'accoutuma à la dépendance vis-à-vis de l'extérieur, si bien que le plan quinquennal de 1982-86 aurait dû être financé à $80 \%$ par l'aide .

600000 à 1000000 de réfugiés affluèrent, chassés par les combats, la collectivisation de l'agriculture et la 'villagisation' en Ethiopie. Pour la plupart, ils se fixèrent dans des camps à la frontière au Nord et au Sud. Au Sud, les sols fertiles et l'eau abondante des deux fleuves offraient des sites d'accueil alors que le Nord soumis à des
5000000 d'habitants, soit une multiplication par plus de quatre ! A.J. Gray fixe le rythme de croissance à 3,3\% par an, soit un doublement en moins de 20 ans. Au début des années 1980, 400000 habitants vivaient à Mugdisho, soit dix fois plus qu'en 1938.

Hormis Berbera et Hargeysa, la plupart des villes sont situées au Benaadir dont le poids démographique s'accroît encore. Selon A.J. Gray, la population urbaine connaît une progression annuelle de $6,5 \%$. Les dernières évaluations antérieures aux combats de 1988, avancent une population de 2000000 d'habitants à Mugdisho qui regrouperait entre les 2/5 et les $2 / 7$ des Somaliens !

Encore aussi nombreux que les agriculteurs et les citadins réunis, il y a dix ans, les éleveurs sont maintenant minoritaires, moins nombreux même que les habitants des villes! Les ventes d'animaux se sont poursuivies à un rythme tel, que la reproduction du troupeau est menacée comme le montre la recrudescence des vols de bestiaux jusqu'au Kenya. Soldats, policiers et fonctionnaires payés irrégulièrement, ont toléré ces pratiques quand ils n'étaient pas complices. Les guerres rituelles entre les clans sont devenues des raids prédateurs!

\section{"Le bateau ivre" du "maire de Mugdisho"12}

Avant de succomber, le président avait joué pour durer, les segments de clansles uns contre autres, amenuisant peu à peu sa "base clanique" et par conséquent sa base territoriale. Il se maintint tant qu'il partagea les entreprises et les revenus de $l^{\prime}$ Etat et redistribua l'aide internationale entre ses fidèles, membres de son clan et de sa propre famille (D. Compagnon). Le népotisme érigé en système de gouvernement et de prédation entraîna une appropriation familiale de l'Etat, sa patrimonialisation. Sa chute brisa même le seul lien qui unissait l'opposition.

Cette dérive maffieuse, cette errance au gré d'alliances de rencontre ${ }^{13}$, acheva de dévaluer l'image de l'Etat comme garant de l'espace économique national et provoqua un repli sur l'espace technique porteur dela reproduction des deux principaux systèmes sociaux de production. Le morcellement actuel du territoire national renvoie à la fois à la segmentation spatiale et à la segmentation sociale où le système clanique fournit des signes de reconnaissance collectifs. N'est-il pas troublant qu'un des romans de Nurrudin Farah s'intitule précisément "Maps" ?

Il est sûr que le partage colonial n'a pas favorisé l'unification d'un espace économique somalien; notamment l'Ogadén entre les mains des Ethiopiens et enfoncé comme un coin entre le Somaliland et la Somalie ex-italienne, a bloqué et ralenti les rapports entre les deux régions. Ce partage recouvrait en dernière analyse, de vieilles divisions 
claniques, religieuses mais séparait également deux systèmes de production dont la logique de la reproduction spatiale conduisait à la mise en oeuvre de dynamiques divergentes. Selon A. Bourgeot, le même processus est à l'oeuvre au Maliet au Niger entrele Nord parcouru par les éleveurs nomades touaregs et le Sud, domaine des agropasteurs et des agriculteurs.

Cette réflexion n'est une prise de position ni dans le débat ni dans le conflit qui opposent les factions, groupes et partis; c'est un appel à la prise en compte des dynamiques spatiales dans la construction du territoire national comme élément de la constitution de l'identité nationale.

\section{Bibliographie}

AFRICA CONTEMPORARY RECORD. ANNUAL SURVEY AND DOCU. MENTS 1987-1988, 1989 "Somali Democratic Republic. The Struggle for Succession", C. Legum, M.E. Doro (eds), New York and London, Africana Publishing Company.

AHMED, C.C. 1989 "God, Anti-colonialism and Drums : Sheykh Uways and the Uwaysiyya", Ufuhamu, 17 (2) : 96-117.

BARDOLPH, J. 1987 "Maps de Nurrudin Farah et l'identité nationale somali", Bulletin des Etudes Africaines de I'INALCO, 7 (13-14) : 249-266.

CASSANELLI, L.V.1982 The shaping of Somali Society : ;Reconstructing the History of a Pastoral People 1600-1900, Philadelphia, University of Philadelphia Press.

DECREANE, P. 1977 L'expérience socialiste somalienne, Paris, Berger-Levraut, Tiers Monde en bref.

GASCON A. 1990 "La Somalie éclatée : crise de régime ou crise d'identité nationale", Islam et sociétés au Sud du Sahara, $n^{\circ} 4$ : 47-55.

GRAY, A.J. Jr.1989 “The Economy of the Somali Democratic Republic in the 1980s", Ufuhamu, 17 (2) : 118-137.

GUIDA D'ITALIA DELLA CONSOCIAZIONE TURISTICA ITALIANA 1938 Africa Orientale Italiana, Milano.

JANZEN, J. 1986 "Ländliche Entwicklung in Somalia. Strukturen, Probleme, Tendezen", Geographische Rundschau, 38 (11) : 557-564.
LAITIN, D.D.; SAMATAR, S.S. 1987 Somalia, Nation in Search of a State, Boulder, Colo., Westview Press, London, Gower.

LEWIS, H.S. 1967 "The origins of the Galla and Somali", Joumal of African History, vol. $7, \mathrm{n}^{\circ} 1$.

LEWIS, I.M.1982 A Modern History of Somalia. Nation and State in the Horn of Africa, Harlow, Longman.

MARKAKIS, J. 1987 National and Class Conflict in the Horn of Africa, Cambridge, Cambridge University Press, Africa Studies Series $n^{\circ} 55$.

MARKAKIS, J. 1989 "The Ishaq-Ogaden Dispute" in A. Hjort aj Ornäs \& M.A.Mohamed Salih (eds), Ecology and Politics, Uppsala, Nordiska Afrikainstitutet.

PROCEEDINGS OF THE 2ND. INTERNATIONAL CONGRESS OF SOMALI STUDIES (Universität Hamburg, Aug. 16, 1983) 1984 Aspects of Development, T. Labahn (ed), vol III.

\section{Communications non encore publiées :}

BOURGEOT, A. "Crise du pastoralisme au Mali et au Niger", exposé au Séminaire des géographes du Laboratoire de Sociologie et de Géographie Africaines (URA 94) et des chercheurs de l'ORSTOM, Paris, 3 avril 1992.

COMPAGNON, D. 1990 "Political Crisis in Somalia : the Legacy of an Exhausted Personnal Rule", paper presented at the Annual Meeting of the African Studies Asssociation, Baltimore, Maryland.

GASCON, A. \& HIRSCH, B. "Naissance $\mathrm{d}^{\prime}$ une frontière. Un siècle de conflit somalo-éthiopien", communication au Colloque "La géopolitique des diplomates, des militaires et des professeurs", organisé par P. Claval, Paris, 26-28 mai 1990.

\section{Notes}

1. Cetarticle reprend le texte remanié de ma communication au Forum : "La guerre civile en Somalie : quand ? comment ? pourquoi ?" organisé par Maxamed Cabdi Maxamed (Association Amitié Franco-Somalienne) à l'Institut du Monde Arabe (Paris 7-8 Avril 1992).

2. Sur ce débat et la conférence prononcée en 1882 par E. Renan en réponse aux historiens allemands (Mommsen) qui justifiaient l'annexion de la province parce que ses habitants parlaient un dialecte germanique, voir les ouvrages suivants :

ZELDIN, T. France 1848-1945, Oxford, Oxford University Press, 1973-1977 (traduction française) Histoire des passions françaises 1848-1945, Paris, Le Seuil Points Histoire, 1980-81.

LE BRAS, H \& TODD, E. L'invention de la France, Paris, Livre de Poche, 1981.

ANCEL,J. Géographie des frontières, Paris, NRF, 1938

3. par analogie avec l'Arabie heureuse.

4. en arabe côte désertique et brâlante.

5. En la débarassant de toute connotation péjorative en revenant au sens originel fort, au sens de Proudhon qui écrivait anarchie : "a-narchie" avec un trait d'union, pour marquer son opposition radicale à toute forme de gouvernement.

6. Isaaq ou Isxaaq, du nom de l'ancêtre éponyme : l'un des six grands clans somali, majoritaire dans l'ex-Somaliland britannique. Ogaadeen: I'un des clans de la grande famille des Daarood, a donné son nom au territoire du même nom, souvent écrit Ogadén selon la graphie éthiopienne (cf. carte).

7. En 1940, face à l'agression italienne, les Britanniques évacuèrent le Somaliland sans combattre et, après la guerre, ils envisagèrentde céderle couloir de Seylac à l'Ethiopie en échange des Reserved Aeras, Hawd et Ogaadeen (A. Gascon \& B. Hirsch).

8. L'Italie reçut le mandat de l'ONU sur son ancienne colonie en 1950, pour dix ans.

9. L'exemple le plus connu est le camp de Tog Wajale proche de la frontière éthiopienne, au Nord.

10. Mouvement connu sous ses initiales anglaises : S.N.M. (Somali National Movement); il exige l'indépendance du Somaliland et a pris le pouvoir à Hargeysa.

11. Ex-Somalie italienne augmentée de l'Ogadén éthiopien mais diminuée du Somaliland.

12. Sobriquet donné à Siyaad dans les deux dernières années de son "règne" alors que ses partisans ne contrôlaient plus que la capitale et ses faubourgs.

13. Aligné sur l'URSS jusqu'à la guerre avec $l^{\prime}$ Ethiopie (1977), Siyaad rejoignit ensuite " 1 'Occident". Le détournement manifeste des subsides dénoncés par la presse ternit l'image du régime qui se tourna alors vers le plus offrant: Arabie Séoudite, Irak, Libye, etc. 